\title{
ANÁLISES DO MATCHING DE IDENTIDADE GENERALIZADO POR CONTINGÊNCIAS DE TRÊS E QUATRO TERMOS: IMPLICAÇÕES PARA EQUIVALÊNCIA DE ESTÍMULOS
}

\author{
Kátia Damiani \\ Maria Amélia Matos (in memoriam) \\ Gerson Yukio Tomanari
}

Resumo: A expansão da tríplice continência em unidades com quatro ou mais elementos abriu novas perspectivas para a compreensão de comportamentos complexos, como a emergência de respostas que derivam da formação de classes de estímulos equivalentes e que modelam comportamentos simbólicos e conceituais. Na investigação experimental, o procedimento de matching to sample tem sido frequentemente empregado para estabelecer discriminações condicionais. Em particular, a obtenção do matching de identidade generalizado é considerada demonstrativa da aquisição dos conceitos de igualdade e diferença. Segundo argumentamos, o fato de se buscar a compreensão desses conceitos a partir de processos discriminativos condicionais pode ter sido responsável pelos frequentes fracassos em demonstrálos em sujeitos não humanos. A falta de correspondência entre os processos discriminativos responsáveis por estabelecer a relação de reflexividade entre estímulos que formam classes equivalentes e o matching de identidade generalizado, nesse sentido, é aqui revista ao longo de estudos empíricos e discutida com respeito às suas implicações.

Palavras-chave: Matching de identidade generalizado. Discriminação condicional. Tríplice contingência. Reflexividade. 
A análise experimental do comportamento tem se dedicado à investigação das variáveis críticas para o estabelecimento de discriminações condicionais. Esse interesse decorre, em grande parte, do fato de a expansão da tríplice contingência ter aberto novas perspectivas para a compreensão de comportamentos complexos, tais como a emergência de respostas que derivam da formação de classes de estímulos equivalentes e modelam comportamentos simbólicos e conceituais (Sidman, 1994).

Para o estabelecimento de uma discriminação condicional, a função discriminativa de um estímulo é determinada pela presença de um quarto elemento, o estímulo condicional. O estabelecimento dessa discriminação, portanto, exige que as funções dos estímulos discriminativos não sejam fixas, uma vez que estas irão se modificar a depender do estímulo condicional presente.

O procedimento majoritariamente empregado para o estabelecimento de discriminações condicionais tem sido o chamado procedimento de "escolha de acordo com o modelo" ou, na língua inglesa, matching to sample. $O$ procedimento de matching, como o denominaremos daqui em diante,é constituído por tentativas nas quais apresenta-se um estímulo, denominado modelo, que deverá exercer a função de estímulo condicional. Normalmente, mediante uma resposta ao modelo, dois ou mais estímulos, denominados comparação, são apresentados e deverão exercer a função de estímulos discriminativos para as respostas de escolha a eles dirigidos e que serão, portanto, diferencialmente consequenciadas.

Para exemplificar, tomemos um treino entre dois conjuntos de estímulos: A (constituído por A1 e A2) e B (constituído por B1 e B2). Os estímulos $A 1$ e $A 2$ funcionam como estímulos modelos e, numa dada tentativa, apenas um deles será apresentado. Os estímulos B1 e B2, por outro lado, estarão presentes simultaneamente como estímulos de comparação em todas as tentativas. Cada um desses estímulos, B1 e B2, poderá funcionar ora como S+ (estímulo na presença do qual respostas são seguidas pela liberação de um reforçador), ora como S- (estímulo na presença do qual respostas encontram-se em extinção), pois suas funções discriminativas dependerão do estímulo modelo presente. Assim, quando A1 estiver presente, B1 será S+ e B2, S-; entretanto, quando A2 estiver presente, as funções se inverterão: no caso, B1 será S- e B2 será S+. Na linguagem técnica, o estímulo modelo é denominado estímulo condicional e a contingência completa de quatro termos é descrita por $\mathrm{S}^{\mathrm{c}}-\mathrm{S}^{\mathrm{d}}-\mathrm{R}-\mathrm{S}^{\mathrm{r}}$.

Entre as possíveis variações do procedimento de matching, a relação entre os estímulos pode ser arbitrariamente definida. No exemplo acima, $A 1, A 2, B 1$ e $B 2$ seriam figuras distintas e configurariam um matching arbitrário ou simbólico. Alternativamente, os estímulos em um procedimento de matching podem ser pareados por suas semelhanças ou diferenças físicas. Seria o caso, no exemplo, de A1 ser um estímulo idêntico a B1 e A2 idêntico a B2. Neste caso, o procedimento é denominado matching de 
identidade. Na situação em que são pareados os estímulos que diferem entre si, tem-se o chamado oddity from sample. Em ambos os casos, tanto o matching de identidade quanto o oddity from sample constituem um matching condicional a partir de contingências de quatro termos.

Cumming e Berryman (1961) e Berryman, Cumming, Cohen e Johnson (1965) exemplificam o uso de procedimentos condicionais com o matching de identidade e o oddity from sample, respectivamente, em pombos. Os autores utilizaram três estímulos: as cores vermelho, verde e azul que iluminavam três discos de respostas alinhados horizontalmente no interior de uma caixa experimental. $O$ estímulo modelo sempre aparecia na chave central e os de comparação eram apresentados nas chaves laterais. No treino de matching de identidade, se $o$ animal bicasse a chave que contivesse o estímulo de escolha idêntico ao modelo, o comedouro ficava disponível por $3 \mathrm{~s}$; se escolhesse o estímulo diferente do modelo, a consequência era 3 s de escurecimento completo da caixa. No treino de oddity from sample a contingência oposta estava em vigor, isto é, se o animal bicasse a chave que contivesse o estímulo de escolha diferente do modelo, o comedouro ficava disponível por $3 \mathrm{~s}$; se escolhesse o idêntico, como consequência ocorria $3 \mathrm{~s}$ de escurecimento completo da caixa. Os três estímulos eram apresentados por igual número de vezes como modelo e cada um deles aparecia como comparação com igual probabilidade na chave à esquerda e à direita do modelo. Ao final do treino, os animais emitiam cerca de $90 \%$ das respostas de escolha que produziam alimento, às quais chamaremos de respostas corretas. Em seguida, os sujeitos foram submetidos a duas sessões de teste durante as quais um estímulo novo, amarelo, era apresentado no lugar do estímulo azul. Ao longo das duas sessões de teste, com exceção da apresentação do estímulo azul, as contingências eram idênticas às de treino. Os resultados revelaram que os animais mantiveram altos índices de respostas corretas nas tentativas idênticas às de treino; entretanto, naquelas em que o estímulo amarelo foi apresentado como $\mathrm{S}+$, o desempenho dos animais foi perturbado. Na vigência do matching de identidade, quando o amarelo foi apresentado como modelo e, portanto, também como comparação S+, o índice de acertos ficou próximo dos 50\% para todos os sujeitos. Nessas tentativas, as respostas de escolha estiveram controladas por posição, ou seja, os sujeitos escolheram predominantemente a chave à direita ou à esquerda do modelo durante toda a sessão. No treino de oddity from sample, nas tentativas em que o amarelo foi apresentado como S+ (e, portanto, não era a cor modelo na tentativa), os desempenhos não se revelaram predominantemente corretos. Em ambos os estudos, portanto, os desempenhos generalizados não foram obtidos. Esses dados foram replicados subsequentemente por outros estudos, tais como Damiani, Passos e Rodrigues (2002), Farthing e Opuda (1974), Zentall, Edwards, Moore e Hogan (1981), entre outros. 
Diferentemente dos infra-humanos, procedimentos que investigam o desempenho de participantes humanos em tarefas de matching de identidade e oddity from sample generalizados têm sido acompanhados por resultados bem-sucedidos. Resultados nesse sentido têm sugerido que humanos respondem com relativa facilidade sob controle de relações generalizadas de identidade e diferença (Brown, Brown, \& Poulson, 1995; Stromer \& Stromer, 1989; Torgrud \& Holborn, 1989), ao contrário das dificuldades que tipicamente acompanham os estudos com infra-humanos. Por exemplo, contando com adaptações especiais nos procedimentos de treino, demonstraram o responder generalizado Galvão, Barros, Lima, Lavratti, Santos, Brino, Dube e Mcllvane (2005), com primatas não humanos, assim como Oden, Thompson e Premack (1988) e Kastak e Schusterman (1994) com leões marinhos.

$\mathrm{Na}$ busca por produzir o responder generalizado de infra-humanos no matching de identidade, os autores desses trabalhos acima citados apontaram as variáveis de treino que consideraram críticas para seus resultados. Oden et al. (1988) atribuíram seus dados positivos ao uso de objetos tridimensionais como estímulos, os quais podiam ser manipulados pelos sujeitos. Galvão et al. (2005) atribuíram seus resultados à utilização de programas de ensino personalizados durante os quais foram instalados e mantidos os pré-requisitos comportamentais necessários para o estudo. Entre esses programas, ressaltaram o treino de discriminações, simples e sucessivas, com repetidas reversões, utilizando os estímulos que, subsequentemente, foram empregados no treino e teste de matching de identidade. Kastak e Schusterman (1994) apontaram a longa e ampla experiência dos sujeitos no matching de identidade em que um grande número de estímulos e relações condicionais com os quais os sujeitos interagiram ao longo de todo experimento.

Para explicar os resultados negativos, Carter e Werner (1978) sugeriram que os desempenhos dos infra-humanos seriam decorrentes do estabelecimento de um dos possíveis controles espúrios: responder por encadeamento ou responder por configuração de estímulos. Segundo os autores, o controle pela relação entre o modelo e os estímulos de comparação constitui um fator necessário para o estabelecimento do desempenho preciso no matching de identidade generalizado. Os autores chamaram esse controle de modelo de regra única, o qual contrasta com os modelos de regras múltiplas e de configuração. O controle por regras múltiplas seria decorrente do estabelecimento de cadeias comportamentais compostas do estímulo modelo, da resposta dirigida ao modelo (frequentemente chamada de resposta de observação), do estímulo de comparação e da resposta de escolha. As regras poderiam ser de dois tipos: regra S+, da qual participaria o comparação correto, e regra S-, da qual participaria o comparação incorreto. $O$ controle por regras múltiplas não resultaria em desempenhos generalizados, pois qualquer alteração na disponibilidade e na sequência 
dos estímulos integrantes da cadeia perturbaria o desempenho. Segundo os autores, os resultados de Cumming e Berryman (1965) e Berryman et al. (1965) poderiam ser interpretados de acordo com regras S+.

O controle de estímulos por configuração seria estabelecido pela unidade formada por cada arranjo de estímulo modelo e comparações laterais que se apresenta aos sujeitos. O responder sob controle das configurações de estímulos também não resulta em desempenhos generalizados, pois qualquer modificação que envolva novas posições dos estímulos de treino ou mesmo a apresentação de estímulos novos deve perturbar o controle de estímulos estabelecido. Na literatura, evidências desse tipo de controle foram encontradas com primatas (Iversen, Sidman, \& Carrigan,1986), ratos (Iversen, 1997) e pombos (Lionello \& Urcuioli, 1998).

Uma proposição teórica que possibilita compreender o papel dos diferentes controles que podem se estabelecer ao longo de um treino em matching to sample diz respeito às Topografias de Controle de Estímulos (TCE). Dube e Mcllvane (1996) propuseram esse termo para se referir às diferentes dimensões dos estímulos que podem adquirir controle sobre o desempenho de qualquer organismo em uma situação experimental qualquer. Essas dimensões, muitas vezes, não são aquelas consideradas relevantes pelo experimentador, mas, por co-variarem com as relevantes, podem estabelecer controle sobre o desempenho do sujeito experimental. Assim, cada um dos controles descritos anteriormente (por regras $\mathrm{S}+\mathrm{e}$ $\mathrm{S}$-, bem como por configurações dos estímulos) constituiria topografias de controle de estímulos diferentes. De acordo com as TCE, qualquer desempenho adventiciamente reforçado na presença de uma dada dimensão de estímulos faz com que essas TCE tenham uma probabilidade diferente de zero de se estabelecer em alguma das tentativas subsequentes.

Avançando na linha de análise das TCEs, seria possível aventar que o estabelecimento do controle condicional pelo estímulo modelo seria uma topografia de controle irrelevante para a geração de um responder generalizado da relação de identidade entre pares de estímulos. A identidade implica uma correlação ponto a ponto entre dois estímulos, ela é uma dimensão discriminativa simples, não condicional. Como qualquer outro treino discriminativo, a identidade deve estabelecer controle sobre o desempenho em decorrência da maior probabilidade de reforçamento na sua presença do que na sua ausência. Dessa forma, são necessários pelo menos três estímulos para que tanto a relação de identidade quanto a de não identidade estejam presentes na situação experimental. No caso do matching de identidade, o experimentador pressupõe que os sujeitos: 1) observem e atentem o estímulo modelo; 2) observem e atentem ambos os estímulos de comparação; 3 ) observem e atentem a relação entre cada comparação e o modelo, e 4) errem e acertem para que o reforçamento diferencial ocorra. Muitas dessas respostas estão fora do controle direto do experimentador, pois as contingências de matching de identidade não 
são explicitamente programadas para instalá-las e mantê-las. Nessas condições, as dificuldades na demonstração do responder generalizado de identidade deveriam ser de fato esperadas.

Corroborando a hipótese de que o estabelecimento de controle condicional seria uma topografia de controle irrelevante, Wright, Cook, Rivera, Sands e Delius (1988) obtiveram desempenhos generalizados na ausência de discriminações condicionais. Os autores empregaram 152 estímulos, de modo que cada um deles aparecia em apenas uma das 76 tentativas de treino. Os estímulos eram apresentados numa tela de computador e os dispensadores de grãos estavam acoplados ao equipamento de tal forma que os grãos eram liberados sobre os estímulos corretos. $O$ teste consistiu da introdução de 10 tentativas por duas sessões consecutivas. Nessas tentativas, 40 estímulos novos foram apresentados, 20 em cada sessão de teste. Os autores conduziram os testes em momentos diferentes do experimento: o primeiro foi realizado quando o desempenho dos animais variava entre $45 \%$ e $60 \%$ de respostas corretas e o segundo quando os sujeitos apresentavam ao menos $80 \%$ de respostas corretas. No primeiro teste, os animais acertaram cerca de $50 \%$ das respostas e, no segundo, cerca de $75 \%$ das respostas. Nesse estudo, portanto, em contraposição à vasta literatura que emprega discriminações condicionais, foi demonstrado o responder generalizado sob controle da semelhança física entre pares de estímulos sob procedimento de matching não condicional.

Aparentemente, o procedimento de Wright et al. (1998) foi capaz de colocar o desempenho do sujeito sob controle da relação de identidade, ainda que o controle por cadeias comportamentais ou por configuração de estímulos possa ter-se estabelecido. Entretanto, para que isso pudesse ocorrer, os sujeitos teriam que aprender 76 cadeias de respostas diferentes e/ou pelo menos 76 configurações diferentes, desempenho muito pouco provável.A propósito, o próprio aumento da quantidade de estímulos pode ter sido suficiente para igualmente gerar desempenhos generalizados no procedimento de matching de identidade condicional. Ou seja, manipulações experimentais adicionais podem vir a aprofundar a compreensão de variáveis em questão. Entretanto, à luz dos resultados de Wright et al.(1988), o procedimento de matching de identidade condicional parece ser bemsucedido no estabelecimento de desempenhos generalizados à medida que promova o estabelecimento do controle discriminativo simples pela relação de identidade entre o modelo e o estímulo de comparação.

\section{Identidade generalizada e equivalência de estímulos}

A busca pelo responder sob controle pela identidade generalizada em procedimento de matching decorre principalmente do fato de esse desempenho ser topograficamente semelhante à reflexividade esperada entre estímulos que compõem uma classe de equivalência. Em um proce- 
dimento típico e bem-sucedido de equivalência de estímulos envolvendo três conjuntos ( $A, B$ e $C$ ) com dois estímulos em cada ( $A 1$ e $A 2, B 1$ e B2, $C 1$ e $\mathrm{C2}$ ), duas classes de estímulos serão estabelecidas (A1-B1-C1 e A2-B2-C2).

Em uma classe de estímulos equivalentes, a demonstração da reflexividade implica a constituição das relações $A-A, B-B$ e C-C, ou seja, a escolha de $A 1$, e não $A 2$, na presença do modelo $A 1$; a escolha de $A 2$, e não de $A 1$, na presença do modelo $A 2$, e assim por diante. Esse desempenho, embora se assemelhe topograficamente ao responder preciso sob matching de identidade generalizado, é resultado de um controle de estímulos diferente: do estabelecimento de classes de equivalência, e não da relação de identidade e diferença entre os estímulos (Sidman \& Tailby, 1982).

A demonstração mais significativa da independência entre a relação de identidade entre estímulos e a reflexividade foi obtida por Johnson e Sidman (1993). Os autores supuseram que, se durante o treino de matching arbitrário fossem estabelecidas relações controladas por S-, ou seja, se o sujeito identificasse o $\mathrm{S}$ - e escolhesse o outro estímulo disponível (controle por rejeição do S-), a demonstração da reflexividade implicaria a escoIha dos estímulos diferentes entre si. Foram realizados treinos A-B, B-C e C-D com dois estímulos de comparação a cada tentativa. Para aumentar a chance de estabelecimento de controle por rejeição, duas condições foram planejadas. A primeira delas foi o procedimento de dica atrasada (delay-cue procedure), no qual, após um intervalo de tempo sem emitir uma resposta de escolha, a chave com o estímulo correto ficava branca e apenas o Spermanecia em exibição; nesse caso, a resposta na chave branca era considerada correta. Esse procedimento foi adotado no início do treino de cada relação condicional. A segunda condição foi o aumento da quantidade de estímulos $\mathrm{S}+\mathrm{e}$ a manutenção de um mesmo $\mathrm{S}$ - nos treinos de cada relação condicional.Assim, por exemplo, no treino da relação A-B, para o modelo A1, os seguintes pares de estímulos de escolha podiam ser apresentados, $\mathrm{B} 1 \mathrm{e}$ $B 2$, ou X1 e B2, ou Y1 e B2; em contraste, quando o modelo era $A 2$, os pares de comparação possíveis eram B2 e B1, ou X2 e B1, ou Y2 e B1. A premissa dos autores era a de que o desempenho que levaria à maior frequência de reforços se daria da forma mais econômica e com menor custo, no caso, por rejeição a S-. Em procedimentos típicos, sem as alterações propostas por Johnson e Sidman (1993), o experimentador esperaria a formação das classes A1B1C1D1 e A2B2C2D2. Entretanto, no procedimento de Johnson e Sidman (1993), foram formadas as classes A1B2C1D2 e A2B1C2D1, em acordo com a possível interpretação de controle por rejeição. Além disso, a previsão de que no teste de reflexividade o sujeito rejeitaria o estímulo idêntico ao modelo e escolheria o outro, o diferente, se confirmou. Com isso, constata-se que a relação de reflexividade encontrava-se sob controle das classes de estímulos estabelecidos e não da relação de identidade entre os modelos e as escolhas correspondentes. 
Conclusivamente, portanto, os resultados disponíveis na literatura sugerem que as dificuldades no estabelecimento do matching de identidade generalizado pode decorrer, em grande parte, da compreensão de que este processo comportamental deva ser analisado à luz de contingências de quatro termos e, por essa razão, deva se servir dos procedimentos de discriminações condicionais. No entanto, resultados bem-sucedidos com pombos procederam com a análise do matching de identidade generalizado em contingências de três termos, nas quais se estabelecem discriminações simples entre a presença e a ausência da relação de identidade e de diferença entre estímulos.

A elucidação das condições necessárias para a demonstração do matching de identidade generalizado com procedimentos condicionais é uma questão relevante per se, mas que, possivelmente, contribuirá apenas indiretamente para a identificação das condições críticas necessárias para a obtenção da equivalência de estímulos com sujeitos não humanos.

\title{
Analyzing generalized identity matching by contingencies of three and four terms: Implications for stimulus equivalence
}

\begin{abstract}
By expanding the three-term contingency into units of four or more elements, new perspectives to comprehend complex behaviors have been opened, such as conceptual and symbolic behaviors modeled by equivalence classes. In the experimental investigation, the matching-to-sample procedure has been employed to establish conditional discriminations. Particularly, a generalized identity matching performance has been considered to demonstrate the acquisition of the concepts of sameness and difference. As we analyze, the concepts comprehension search, based on conditional discriminations, has been responsible for the failures to demonstrate them in non-human subjects. In this paper, the distinctions between the discriminative processes that lead to a reflexivity performance in an equivalence class and the generalized matching to sample is examined and discussed.
\end{abstract}

Keywords: Generalized identity matching. Conditional discrimination. Triple contingency. Reflexivity. 
Analyses de appariement de ègalitè (identity matching) généralisée via trois et quatre terms: implication pour le stimuli équivalence

Résumé: L'élargissement de la triple contingence en unités avec quatre ou plus termes ouvrit nouvelles pespectives pour la compréhension de comportements complexes comme l'émergence de réponces provenant de l'établissement de classes de stimuli équivalentes qui à son tour modèlent comportements symboliques et conceptuelles. Dans la recherche expérimentale, le procédure de appariement de ègalite a été utilisé fréquemment pour établir des discriminations conditionelles. Particulièrement, la obtention de l'égalite généralisée est considere comme une manifestation des concepts d'égalité et de différence. Selon le texte actuel, le fait de chercher à comprendre ces concepts à partir de procédures de discrimination conditionnelle peut avoir été responsable des échecs fréquents avec des animaux (non humains). L'absence de corrélation entre les processus responsables de l'établissement les classes d'équivalence les stimuli et l'égalité généralisée est discutée sur la base des résultats d'études empiriques et ses implication.

Mots clés: Appariement de ègalitè. Stimuli équivalence. Triple contingence. Discriminations conditionelles.

Análisis de la igualación de identidad generalizada por contingencias de tres y cuatro términos: implicaciones la equivalencia de estímulos

Resumen: La expansión de la triple relación de contingencia en unidades con cuatro, o más, elementos abrió nuevas perspectivas para la comprensión de comportamientos complejos, tal como la emergencia de respuestas que derivan de la formación de clases deestímulos equivalentes y que modela comportamientos simbólicos y conceptuales. En la investigación experimental, el procedimiento de Igualación a la Muestra (Matching to Sample o MTS en inglés) se ha empleado frecuentemente para establecer discriminaciones condicionales. En particular, la obtención de igualación de identidad generalizada es considerada como una demostración de la adquisición de los conceptos de igualdad y diferencia. Según argumentamos, el hecho de intentar comprender esos conceptos a partir de procesos de discriminación condicional puede haber sido responsable por los frecuentes fracasos cuando se ha intentado encontrar en sujetos no humanos. La falta de correspondencia entre los procesos de discriminación responsables por establecer la relación de reflexividad entre estímulos que forman clases equivalentes y la igualación de identidad generalizada, en ese sentido, es revisada aquí a lo largo de estudios empíricos y son discutidas sus implicaciones.

Palabras clave: Igualación de identidad generalizada. Discriminación condicional. Triple contingencia. Reflexividad. 


\section{Referências}

Berryman R., Cumming, W. W., Cohen, L. R., \& Johnson, D. F. (1965). Acquisition and transfer of simultaneous oddity. Psychological Reports, 17, 767-775.

Brown, A. K., Brown, J. L., \& Poulson, C. L. (1995). Generalization of children's identity matching-to-sample performances to novel stimulus. The Psychological Record, 45, 29-43.

Carter, D. E., \& Werner, T. J. (1978). Complex learning and information processing by pigeons: A critical analysis. Journal of Experimental Analysis of Behavior, 29, 565-601.

Cumming, W. W., \& Berryman, R. (1961). Some data on matching behavior in the pigeon. Journal of the Experimental Analysis of Behavior, 4, 281-284.

Cumming, W. W., \& Berryman R. (1965). The complex discriminated operant: Studies of maching-to-sample. In D. I. Mostofsky (Ed.), Stimulus generalization (pp. 284330). Stanford, CA., Stanford University Press.

Damiani, K., Passos, M. L. R. F., \& Matos, M. A. (2002). Seqüência de estímulos durante o fortalecimento da resposta de bicar: efeitos sobre a aquisição de desempenhos em matching e oddity. Psicologia: Reflexão e Crítica, 15, 465-473.

Dube, W. V., \& Mcllvane, W. J. (1996). Implications of a stimulus control topography analysis for emergent behavior and stimulus classes. In T. R. Zentall \& P. M. Smeets (Eds.), Stimulus class formation in humans and animals (pp. 197-222). The Netherlands: Elsevier Science B.V.

Farthing, G. W., \& Opuda, M. J. (1974). Transfer of matching-to-sample in pigeons. Journal of Experimental Analysis of Behavior, 21, 199-213.

Galvão, O. F., Barros, R. S., Lima, S. B., Lavratti, C. M., Santos, J. R., Brino, A. L. F., Dube, W. V., \& Mcllvane, W. J. (2005). Extent and limits of the matching concept in Cebus apella: A matter of experimental control? The Psychological Record, 55, 219-232.

Iversen, I. H. (1997). Matching-to-sample peformance in rats: A case of mistaken identity? Journal of The Experimental Analysis of Behavior, 68, 27-45.

Iversen, I. H., Sidman, M., \& Carrigan, P. (1986). Stimulus definition in conditional discriminations. Journal of the Experimental Analysis of Behavior, 45, 297-304.

Johnson, C., \& Sidman, M. (1993). Conditional discrimination and equivalence relations: Control by negative stimuli. Journal of the Experimental Analysis of Behavior, 59, 333-347. 
Lionello, K. M., \& Urcuioli, P. J. (1998). Control by sample location in pigeons'matching to sample. Journal of the Experimental Analysis of Behavior, 70, 235-251.

Oden, D. L., Thompson, R. K. R., \& Premack, D. (1988). Spontaneous transfer of matching by infant chimpanzees. Journal of Experimental Psychology: Animal Behavior Processes, 14, 140-145.

Kastak, D., \& Schusterman, R. (1994). Transfer of visual identity matching-to-sample in two California sea lions (Zalophus californianus). Animal Learning e Behavior, 22, 427-435.

Sidman, M. (1994). Equivalence Relations and Behavior: A research story. Boston: Authors Cooperative.

Sidman, M., \& Tailby, W. (1982). Conditional discrimination vs. matching to sample: An expansion of the testing paradigm. Journal of the Experimental Analysis of Behavior, 37, 5-22.

Stromer, R., \& Stromer, J. B.(1989). Children's identity matching and oddity: Assessing control by specific and general sample-comparison relations. Journal of the Experimental Analysis of Behavior, 51, 47-69.

Torgrud, L. J., \& Holborn, S. W. (1989). Effectiveness and persistence of precurrent mediating behavior in delayed matching to sample and oddity matching with children. Journal of the Experimental Analysis of Behavior, 52, 181-191.

Wright, A. A., Cook, R. G., Rivera, J. J., Sands, S. F., \& Delius, J. D. (1988). Concept learning by pigeons: Matching-to-sample with trial-unique video picture stimuli. Animal Learning \& Behavior, 16, 436-444.

Zentall, T. R., Edwards, C. A., Moore, B. S., \& Hogan, D. E. (1981). Identity: The basis for both matching and oddity learning in pigeons. Journal of Experimental Psychology: Animal Behavior Processes, 7, 70-86.

Kátia Damiani, Instituto de Psicologia, Universidade de São Paulo. Av. Prof. Mello Moraes, 1721.CEP:05508-030, São Paulo, SP. Endereço eletrônico: katia@starplast.com.br

Gerson Yukio Tomanari, Professor Doutor do Instituto de Psicologia da Universidade de São Paulo. Av. Prof. Mello Moraes, 1721. CEP: 05508-030, São Paulo, SP. Endereço eletrônico: tomanari@usp.br

Recebido em: 22/03/2010

Aceito em: 25/05/2010 
